\title{
Heuristic procedures for a stochastic lot-sizing problem in make-to-order manufacturing
}

\author{
N.P. Dellaert and M.T. Melo* \\ Econometric Institute, Erasmus University Rotterdam. \\ Rotterdam, The Netherlands
}

We consider a single item, uncapacitated stochastic lot-sizing problem motivated by a Dutch make-to-order company producing steel pipes. Since no finished goods inventory is kept, a delivery date is fixed upon arrival of each order. The objective is to determine the optimal size of production lots so that delivery dates are met as closely as possible with a limited number of set-ups. Orders that are not satisfied on time are backordered and a penalty cost is incurred in those cases. We formulate the problem as a Markov Decision Process and determine the optimal production policy by dynamic programming. Since this approach can only be applied to very small examples, attention is given to the development of three simple lot-sizing rules. The first strategy consists of producing the orders for a fixed number $T$ of periods whenever the demand for the current period reaches a pre-specified limit $x$. A simple set of tests is proposed leading to cost improvements in situations where the best combination for the decision variables $x$ and $T$ deviates from the optimal policy. The second lot-sizing rule is based on the wellknown Silver-Meal heuristic for the case of deterministic time-varying demand. A fixed cycle production strategy is also derived. Numerical examples taking into account different demand patterns are provided. The analysis of the results suggests that the first heuristic is particularly suitable for the problem under consideration. Finally, the model is incorporated in the operations control level of the hierarchical production planning system of the Dutch company and assists the management in the evaluation of the quality of the aggregate decisions. A consequence of this feedback mechanism is the modification of the aggregate plans.

\section{Introduction}

In recent years, an increasing number of companies in the manufacturing industry have oriented their production to the make-to-order (MTO) sector. Mattsson et al. [18] report the results of a study of fifty-nine Swedish companies during seven years where $50 \%$ of the companies increased the degree of MTO. The authors observed that $37 \%$ of the companies dedicated $100 \%$ of the production to the MTO sector,

\footnotetext{
${ }^{\star}$ On leave from D.E.I.O. (Universidade de Lisboa, Portugal). This research was supported by J.N.I.C.T. (Portugal) under contract BD/2264/92.IA.
} 
while in $46 \%$ the level was between $50 \%$ and $95 \%$. Only nine companies remained in the make-to-stock (MTS) sector. In the United Kingdom, a survey conducted by Tobin et al. [26] showed that there were about 2000 small and medium sized MTO companies equally split between component subcontractors and producers of capital equipment.

MTO companies manufacture products designed specifically to meet the needs of each individual customer rather than standard products supplied from stock. Hendry and Kingsman [11] establish the main differences between MTO and MTS companies. As stressed by these authors, in the MTO environment due to a com-petitive market both the delivery time and the price quoted for a particular job play a vital role in promoting customer satisfaction and generating future business. Failing to meet a promised delivery date may result in lost profit and lost market share. Kingsman et al. [15] present a methodology to integrate production and marketing considerations for quoting for orders. They acknowledge a common situation where the sales/marketing department quotes delivery dates and prices which maximize the chance of the company winning an order but disregard the ability of the production to respond to the promised delivery dates. Essentially, the approach proposed by Kingsman et al. consists of estimating the probability of winning an order. This probability is up-dated each time the result of a quotation to a customer in a certain category is known.

Once the jobs are accepted, production planning takes place. In Hendry and Kingsman [12], a job release mechanism is described to control the shop floor throughput times so that delivery dates can be met without large deviations.

Production planning models for the MTS area have received considerable attention in the literature. As a consequence, an extensive collection of results is available. We refer to Silver and Peterson [24] and Graves et al. [9] for comprehensive reviews of models and methods developed both for the deterministic and the stochastic demand situations. Unfortunately, the MTO area has received much less attention. Hendry and Kingsman [11] claim that well-known planning approaches for the MTS sector like MRP, MRP II, OPT and JIT do not address the special characteristics and needs of MTO systems and therefore can not be applied in practice. Concerning the applicability of MRP, the delivery date negotiated with the customer for an order determines the placement of the order in the master schedule. MRP logic then plans the necessary production. In the MTO sector, the master schedule is comprised of plans for final assembly and delivery to customers. At least two case studies were found in the literature where the methodology of MRP seemed to have been applied with a claim of success to an MTO company. Hoey et al. [13] report on the design of the key elements of an MRP system applied effectively to the MTO sector. They illustrate their approach within a low-volume company manufacturing corrugator equipment. In a later paper (McAreavey et al. [19]), these ideas are further developed and a closed loop MRP is proposed considering rough cut capacity planning. The authors refer to substantial reductions in work-in-progress and manufacturing lead times by using their techniques. 
Other production approaches for MTO were also explored. These include an integer goal programming model especially designed by Markland et al. [17] for a US producer of tapes, and a Decision Support System (DSS) developed by Lecomte and Dejax [16] for the production control of an MTO manufacturer of abrasive products. The latter system is generated on a Knowledge Based DSS development tool and integrates a scheduling heuristic that provides a compromise between minimizing the times for changing tools and minimizing the delays in satisfying the orders. Another description of a DSS can also be found in Hendry [10], where an attempt to manage delivery and manufacturing lead times is made.

The purpose of this paper is to model an MTO situation as simply as possible to enable quantitative analysis of production rules. In contrast with most of the case studies mentioned above, where simulation is used to evaluate the performance of the planning rules, we give special emphasis to the mathematical analysis. To the best of our knowledge, only methods based on queueing theory have been subject to a mathematical analysis. Graves et al. [9] provide an extensive survey on queueing models for production systems, while Dellaert [4] reports on an application of queueing theory to MTO. Our model was largely inspired by a lot-sizing problem arising in a Dutch MTO company producing welded steel pipes. In section 2, we describe the problem in detail. In order to cope with the uncertainty regarding the amount as well as the time of receipt of the orders, we model the problem as a Markov Decision Process with discrete state and time space. This is presented in section 3 . In section 4, focus is given to an algorithm to obtain an optimal production policy. Since in most practical situations finding an optimal solution is computationally too complex, we concentrate on the design of heuristic procedures. Section 5 is devoted to the development of three different production strategies. In section 6, the numerical results obtained for some test problems are presented. For a very simple demand distribution, the optimal average costs are computed and compared to the average costs obtained with the heuristics. Further results are presented for more realistic demand distributions. Section 7 contains our main conclusions and directions for further research.

\section{The problem}

Wagner and Whitin [28], in a classic article, developed an efficient dynamic programming algorithm for solving a finite horizon, lot-sizing problem. The algorithm applies to the case of deterministic time-varying demand where backordering is not allowed. These two features of the Wagner-Whitin model are difficult to fulfill in many practical settings. Our main objective is, by starting with the problem addressed by the Wagner-Whitin algorithm, to design a model capable of capturing some of the elements of an MTO situation, namely, the demand uncertainty and complete backordering. Our study was motivated by a situation observed by a Dutch manufacturer of welded steel pipes. The production process consists of a preliminary treatment, the welding process and a follow-up treatment. The bottleneck in the production process 
appears to be the welding process. There are several parallel welding machines, each with a different range of sizes. Whenever a certain type of steel pipe is produced, parts of the welding machine have to be rebuilt. As a result, the steel pipes are divided into several groups of different sizes among which the rebuilding times are small. To avoid too much loss of time for set-ups, the usual procedure followed by the company consists of producing steel pipes belonging to the same type consecutively on the same machine.

The demand for the steel pipes can not be predicted with a high level of certainty because the company does not know if and when a customer places an order. Due to the demand uncertainty together with the products' high volume and customer-specific nature, no finished goods inventory is kept.

In order to meet each customer's specific requirements, the company manufactures the steel pipes on an MTO basis and agrees upon a certain delivery date for each incoming order.

Clearly, the nature of the process just described makes production planning in this situation very difficult. To gain some insight into the problem, some elements were relaxed or simply disregarded in a preliminary study. This consisted in developing a pilot scheme for the lot-sizing of a single type of product which is manufactured on a single machine without capacity limitations. Lee and Nahmias argue in [9] that most single-product models are able to capture the most important features of a problem, so that explicit inclusion of the interaction of different items is not necessary. In particular, if capacity restrictions are rather unimportant, then one does not need to consider all product types together. Moreover, if many products have to be dealt with simultaneously (this corresponds in our case to about 150 items of different shapes), a multiple-product model is often unmanageable and an optimal solution is impossible to derive, at least from a practical point of view. Reasons for these difficulties are discussed by Axsäter [1]. On the other hand, although capacity constraints are excluded from our model, capacity requirements can be reflected in the set-up costs, which means that the model takes into account that small lots of orders require relatively more capacity than large lots. Also, one can argue that applying an uncapacitated model and then trying to adjust the resulting production schedules may be possible in practice. An example of such a case is given by Van Nunen and Wessels [27]. For the classical single-item uncapacitated dynamic lot-sizing problem with an infinite time horizon, these authors developed a simple procedure for obtaining a production schedule using an extension of the Wagner-Whitin algorithm [28]. The application of their procedure to each product independently usually results in an infeasible solution in one or more of the production periods, since the capacity constraints are violated. Therefore, in a second stage a capacity adapting procedure is used. This consists of shifting the production of whole series of products from some period $t$ to an earlier period $t-k$ if there is capacity available in period $t-k$ and a shortage in period $t$. Such a shift is only made if the hiring of extra capacity in period $t$ is more expensive than the extra holding costs caused by the shifting. The selection of 
a series to be shifted is based upon its costs savings per unit of capacity. In the situations investigated by Van Nunen and Wessels [27] at a major Dutch company, savings on set-up and holding costs of $20 \%$ were achieved in comparison with the planning procedures used by the company until then.

Decisions concerning the production of the orders are assumed to be taken periodically. Most stochastic inventory models adopt this review process. Silver and Peterson [24] state the advantages of periodic review as giving lower reviewing costs and allowing a reasonable prediction of the level of the workload on the staff involved in issuing replenishment orders. Periodic review also makes the description of the state of the system much simpler and thereby the analysis. The length of the review period is fixed and the decisions can be made only at predetermined times corresponding to the end of the periods.

Following the wishes of the management of the steel company, orders are divided into different categories varying from very urgent orders to orders with a requested delivery date of a few months. In addition, the company is engaged with a regular number of customers with whom long-term agreements are established concerning the assignment of the delivery dates on receipt of the orders. Due to these elements, it seems reasonable to assume that the customers are classified in $N$ different groups. The mechanism of delivery date assignment consists of offering each customer a fixed lead time so that all customers belonging to a group $i(1 \leq i \leq N)$ obtain a lead time equal to $i$ periods when they place an order. This means that in an arbitrary period $t$, the company promises customers of group $i(1 \leq i \leq N)$ to have their orders ready by the end of period $t+i$. Seidmann and Smith [22] and Quaddus [21], among others, investigated models that assign a constant delivery date to every order. The purpose of such models is to find an optimal lead time which can be quoted for all incoming orders. Clearly, due to the nature of our problem and the requirements of the customers, this approach does not seem appropriate in our case.

A further assumption in our model is that the probability distribution of the demand for the next $N$ periods is known, stationary and independent of the arrival period of the orders. This supposition stems from the long-term agreements established with the customers. On the other hand, it makes it possible to give a mathematical steady-state analysis of the problem. Furthermore, it is also assumed that the demand of the different groups of customers is stochastically independent.

Every order placed by a customer consists of one unit of product. Customer demands that are not available at the promised delivery dates are backordered. Cruickshanks et al. [3] discuss a production smoothing model for an MTO company for which the market requires a firm delivery time since the customers do not tolerate late delivery due to their own tight production schedules. Cruickshanks et al. introduce a so-called planning window, which represents the amount by which the promised delivery date exceeds the production lead time for a given product. The authors also derive an approximate analytic model for which backordering is allowed. From a simulation study for normally i.i.d. and stationary demand, the authors conclude that 
significant smoothing benefits can be achieved by using a planning window. Moreover, the approximate model has some predictive value with regard to the behaviour of the first model.

An interesting feature of MTO with fixed lead times is that a gradually decreasing knowledge about the future required deliveries can be observed. This means that by the end of each review period $t$, the exact number of orders for period $t+1$ is known but only partial information is available on the demand for the following $N-1$ periods because of the possibility of receiving more orders for those periods.

By considering the demand distribution and the orders known at the end of a review period $t$, one of the following decisions can be made:

(i) delay production;

(ii) produce all known orders;

(iii) produce a subset of the known orders.

The selection of the first decision results in not being able to meet the demand for period $t+1$ on schedule. Consequently, a penalty cost $p>0$ is charged to each late order. This provides an incentive to manufacture orders on time. Selecting decisions (ii) or (iii) yields set-up costs and also holding costs. Like in many other traditional lot-sizing formulations, we assume a fixed set-up cost $s$ which accounts for the preparation of the machine before the production of a lot is initiated. The parameter $s$ may also comprise administrative costs, preparation costs, etc. Large set-up costs are usually involved in the company of our case study. Furthermore, a holding cost is incurred at rate $h>0$ per period for each order that is finished before its delivery date. This cost is charged because a lot of customers find it inconvenient to have untimely deliveries. Usually, the holding cost per order will be considerably smaller than the backorder penalty cost. Our objective is to find a proper scheduling of the production so that the delivery dates are met as closely as possible at the expense of minimal average costs per review period.

In the next section, the details of the model proposed for our problem are presented.

Before concluding this section, we briefly refer to the usefulness of our model. Although in many complex practical situations it may not be directly applicable due to the simplifying assumptions, it can be seen as a starting point. We believe it can provide useful insights into the form of a solution for the original problem. In addition, the impact of the delivery dates fixed by the management can be examined not only in terms of costs and size of the lots, but also from the customers' perspective. This means that important issues affecting customer satisfaction, such as the number of late orders, can also be analysed. Furthermore, the model can be integrated in an hierarchical production planning environment. After applying to each product the production rules that will be discussed in section 5 , the manager is apt to decide which product should be manufactured on which machines and use a capacity adapting 
procedure of the type suggested by Van Nunen and Wessels [27] in order to comply with capacity restrictions. In the company of our case study, our model was incorporated in the lowest level of the hierarchical production process and helped the management to assess the quality of aggregate decisions.

\section{Model formulation}

The assumptions made in our problem enable us to consider a Markov Decision Process (MDP) with discrete state and time space (Tijms [25]). At each decision point, a state is observed and an action is chosen. A state is defined as an $\mathrm{N}$ component vector where each component represents the required deliveries for a certain period. The observation of an order state vector $r=\left(r_{1}, r_{2}, \ldots, r_{N}\right)$ at the end of period $t$ gives the following information:

$$
r_{i}= \begin{cases}\text { number of orders of unit size to be delivered during } \\ \text { period } t+1, \text { including backorders from earlier periods } & \text { if } i=1, \\ \text { number of orders of unit size to be delivered during } & \\ \text { period } t+1 & \text { if } 2 \leq i \leq N .\end{cases}
$$

We denote the set of all possible states by $R$.

Each state $r \in R$ is associated with a non-empty set of actions $A(r)$. Since the available capacity is unlimited and the opportunities for production occur at discrete points, the best strategy must involve manufacturing quantities that last for an integer number of periods like in the deterministic dynamic version of our lot-sizing problem (see Zangwill [29] and Denardo and Lee [7]). As a result, an action $a$ specifies the amount of periods for which the corresponding known orders are produced, i.e. it determines the size of a lot as being equal to $\sum_{i=1}^{a} r_{i}$. In particular, the action $a=0$ specifies that production is delayed. The action space $A(r)$ is defined for every state $r \in R$ as

$$
A(r)=\mid \begin{array}{ll}
\{0\} & \text { if } r_{1}=0 \\
\{1, \ldots, N\} & \text { if } r_{1} p>s \\
\{0,1, \ldots, N\} & \text { otherwise }
\end{array}
$$

Observe that if the penalty costs are larger than the set-up costs, then it is clearly not optimal to delay the production.

If we have taken action $a$ in state $r$, let $Q_{a}(r)$ denote the state at the end of the next period, just before the new orders are added to the order state. To illustrate the meaning of $Q_{a}(r)$, suppose four groups of customers place orders and at the end of period $t$ a certain state vector $r=\left(r_{1}, r_{2}, r_{3}, r_{4}\right)$ is observed. If production is delayed, then at the end of the following period we have $Q_{0}(r)=\left(r_{1}+r_{2}, r_{3}, r_{4}, 0\right)$. Similarly, $Q_{3}(r)=\left(0,0, r_{4}, 0\right)$. 
Let $J \subseteq \mathbb{N}^{N}$ denote the set of all possible required deliveries $\left(j_{1}, j_{2}, \ldots, j_{N}\right)$ corresponding to the orders arriving during one period for the next $N$ periods. Thus, if action $a \in A(r)$ is taken on observing state $r$, we enter a state $z$ given by $z=Q_{a}(r)$ $+\left(j_{1}, j_{2}, \ldots, j_{N}\right)$. Moreover, let $d_{i j}$ be the known probability that during an arbitrary period $t$, a total number of $j$ units of product is ordered by customers of group $i$ for period $t+i$. The transition probability $P_{r z}^{a}$ of moving from a state $r$ to a state $z$ by choosing action $a \in A(r)$ is therefore defined by

$$
P_{r z}^{a}= \begin{cases}\prod_{i=1}^{N} d_{i j_{i}} & \text { if }\left(j_{1}, j_{2}, \ldots, j_{N}\right) \in J, \\ 0 & \text { otherwise. }\end{cases}
$$

Finally, the costs associated with action $a \in A(r)$ are given by

$$
q_{r}^{a}= \begin{cases}p r_{1} & \text { if } a=0, \\ s+h \sum_{i=1}^{a-1} i r_{i+1} & \text { if } a>0 .\end{cases}
$$

Note that $p r_{1}$ is the cost incurred by decision (i), while the remaining costs correspond to decisions (ii) and (iii).

Given the Markov Decision Process described above, our goal is to find an optimal production policy. The optimality criterion is to minimize the long-run average costs per period. In the next section, we will focus on this point.

In Ten Kate [14], a similar model to ours is discussed. The author considers multiple products that are spread over a small number of families and produced on a single machine. Contrary to our case, the same lead time is assigned to all families. Moreover, both set-up times and processing times are taken into account with respect to the derivation of two strategies for the problem of order acceptance.

\section{The optimal production policy}

By the choice of the action space, all stationary policies have transition probability matrices representing recurrent aperiodic Markov chains. If the number of possible states is limited, i.e. if the one-period demand is bounded, the optimal production policy can be determined by using a policy iteration method. However, since the method of successive iteration as described by Odoni [20] performs faster in our situation, it will be preferred in this case. We are interested in finding the policy that minimizes the expected costs $g$ per transition. If $v_{n}(r)$ is defined as the minimum total expected costs from the next $n$ transitions when the current state is $r$ and an optimal policy is followed, the iteration scheme takes the form described in the optimality principle by Bellman [2]:

$$
v_{n+1}(r)=\min _{a \in A(r)}\left[q_{r}^{a}+\sum_{z \in R} P_{r z}^{a} v_{n}(z)\right], \quad r \in R, n=0,1, \ldots,
$$

with $P_{r z}^{a}$ and $q_{r}^{a}$ given by (1) and (2), respectively. 
Observe that it is not necessary to consider all possible values for the first component $r_{1}$ of the order state vector. If for some state $r \in R$, action $a>0$ is optimal, then the same action will also be optimal for all states $\left(y, r_{2}, r_{3}, \ldots, r_{N}\right)$, with $y>r_{1}$ because $r_{1}$ is not involved in the holding costs.

By applying the results of Odoni [20], it can be proved that any production rule achieving the minima in (3) also has minimal costs per transition. Therefore, starting with $v_{0}(r)=0$ for every $r \in R$, the computation of (3) is repeated until a satisfactory degree of convergence is achieved. This provides lower and upper bounds on the minimal average costs $g$, which we denote by $L_{1}(n)$ and $L_{2}(n)$, respectively. It follows that $g$ may be estimated as $\left[L_{1}(n)+L_{2}(n)\right] / 2$. This estimate becomes nearly exact for large $n$.

Although the above technique is commonly used for solving an MDP with an infinite planning horizon (see Tijms [25]), from a practical point of view its applicability is limited due to the extremely fast growth of storage (and time) requirements as the dimension of the state space increases. Computation times become prohibitively large even for small problems. For this reason, it is desirable to derive good heuristics, that is, fast lot-sizing rules with relatively low average costs per period. In the next section, we present three different production strategies.

\section{Heuristic procedures}

It is generally agreed that in practical settings the use of simple and intuitive procedures to solve lot-sizing problems is preferred to more complex algorithms. An example of a method that has received limited acceptance in practice is the WagnerWhitin algorithm [28], although is guarantees an optimal selection of replenishment quantities for the uncapacitated dynamic lot-sizing problem. The main reasons for this lack of acceptance are the relatively complex nature of the algorithm which makes its understanding difficult for managers, and the considerable computational effort required. In addition, the non-realistic nature of the demand also accounts for the low receptivity of the algorithm.

In this section, we focus on the design of three simple lot-sizing rules. By combining orders into production lots, we can save on set-up costs at the expense of some additional holding and penalty costs. The heuristics that are proposed differ in the way in which they combine demands into lots.

We start by giving a detailed description of a simple strategy called the $(x, T)$ rule. This rule consists of producing the known orders for the next $T$ periods if the demand for the current period is at least $x$ units. Cost improvements are achieved with refinements of the rule, which are discussed in subsection 5.1.1. In subsection 5.2 , we present a production strategy based on the well-known Silver-Meal heuristic (Silver and Meal [23]) for the deterministic version of our problem. Finally, subsection 5.3 deals with a fixed cycle production rule. Before giving a description of the three heuristic procedures, we introduce the notation that will be used. 
Let $X_{t, t+i}$ denote the number of orders that arrive during period $t$ to be delivered by the end of period $t+i$, with $i=1,2, \ldots, N$. In other words, $X_{t, t+i}$ contains the demand placed by customers of group $i(1 \leq i \leq N)$ during period $t$. In figure 1 , an illustration of the meaning of these variables is presented. Note that all demand for a certain period is found in the same column.

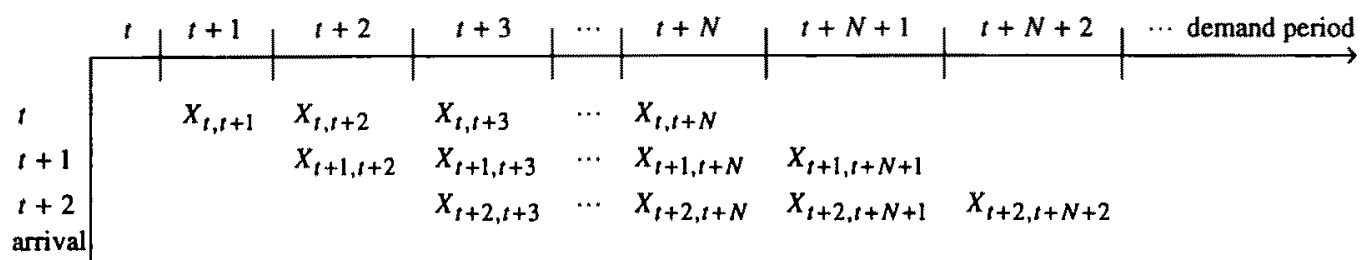

Figure 1. Demand for various periods.

The expectation of the random variable $X_{t, t+i}$ is denoted by $u_{t, t+i}$ and due to the assumption of stationary demand is determined by

$$
u_{t, t+i}=\mathcal{E}\left(X_{t, t+i}\right)=\sum_{j>0} j d_{i j}=u_{i} .
$$

If we define $b_{i \ell t}$ as the probability that during the last $i$ periods before an arbitrary period $t$, customers order a total number of $\ell$ orders for period $t$, it follows that $b_{i \ell t}=\mathcal{P}\left\{\sum_{n=t-i}^{t-1} X_{n t}=\ell\right\}$. Let $J_{i \ell}$ be the set of all possible one-period demands $\left(j_{1}, j_{2}, \ldots, j_{N}\right)$ for which the sum of the first $i$ components equals $\ell$. Since demand is stationary, we obtain (observe figure 1)

$$
b_{i \ell t}=\sum_{J_{i \ell}} \prod_{k=1}^{i} d_{k j_{k}}=b_{i \ell} .
$$

Finally, $e_{i t}$ is defined as the expected value of $r_{i}$ (i.e. the $i$ th component of the state vector $r$ ) at the end of period $t$, if no required deliveries for period $t+i$ have been produced, that is,

$$
e_{i t}=\mathcal{E}\left(\sum_{n=t+i-N}^{t} X_{n, t+i}\right)=\sum_{n=t+i-N}^{t} u_{n, t+i}=\sum_{m=i}^{N} u_{m}=e_{i} .
$$

\subsection{THE $(x, T)$-RULE}

While testing the optimal production policy for several examples, we noticed that in most cases the decision whether to produce or not depends only on the value of $r_{1}$ and not on the required deliveries for later periods. Moreover, for a given demand distribution, the number of periods for which orders are produced is nearly 
always the same. Only in "extreme" states with a rather small probability does the optimal policy deviate from these results. The form of the optimal policy is explained by the interaction between the cost parameters and the demand pattern. Since the setup costs are considerable, the production is delayed as much as possible. In most situations, the value of $r_{1}$ alone determines when a set-up should take place. Observe that in the short term the most important orders are those that have to be delivered by the end of the next period. Failing to meet them yields penalty costs and if one waits too long, these costs will become larger than the set-up costs. Recall that whenever $r_{1} p>s$, the action space starts with action $a=1$, meaning that postponing production will certainly be too expensive. With respect to the number of periods for which the known orders are manufactured, both the holding costs and the demand pattern play an important role. Producing the demand for more periods increases the holding costs, but if the known amount is large enough it will be cheaper to produce it now than to produce a smaller amount and take the next set-up sooner. On the other hand, our knowledge about the future orders decreases with the number of periods and so it may be better not to produce the known demand for periods that are further away from the current one.

Based on the results of the tests performed, we developed a simple decision rule which only uses the information about the number of orders for the first period and the number of backorders, i.e. the value of $r_{1}$. If $r_{1}$ is smaller than some decision variable $x$, there is no production during the first period. Otherwise (i.e. if $r_{1} \geq x$ ), all known orders with a residual lead time of $T$ periods or less are produced during that period. Like $x, T$ is also a decision variable. We call this production strategy the $(x, T)$-rule. Since a decision concerning the production is made at the end of every period, it follows that production may take place less than $T$ periods after the previous one if in the meantime enough orders arrive.

One of the main advantages of the $(x, T)$-rule is that the average costs per period can easily be determined for given values of $x$ and $T$. In order to compute these costs, we consider the order state during one production cycle. Since demand is stationary and the $(x, T)$-rule is a fixed production strategy, there exist time points at which the stochastic process that represents the order state probabilistically restarts itself. From the coming analysis, it will be clear that one of these regenerative points takes place at the end of a period in which the required deliveries for the first $T$ periods have been produced. Since the probabilities for each possible order state vector are the same in every regenerative point, the average costs per period are determined by considering the order state during the periods between two regenerative points, i.e. between two consecutive production periods.

Although $r=\left(r_{1}, r_{2}, \ldots, r_{N}\right)$ provides all necessary information about the order state, it is not suitable for the analysis of situations in which the dimension of the state space is very large. On the other hand, in order to make a decision by following the $(x, T)$-rule, it is sufficient to know the value of $r_{1}$. For these reasons, we replace the order state vector $r=\left(r_{1}, r_{2}, \ldots, r_{N}\right)$ by $r_{1}$. However, $r_{1}$ does not provide the 
necessary information about the transition probabilities. The changes in the $r_{1}$ value depend on the time passed since the last production period. We will illustrate this by means of an example. Suppose there are four groups of customers and $T=3$. At the end of an arbitrary period $t-1$, we observe that $r_{1} \geq x$. As a result, all orders with a delivery date less than or equal to $t+2$ are produced during period $t$. At the end of period $t$, the value of $r_{1}$ is updated with the value of the stochastic variable $X_{t, t+1}$, i.e. $r_{1} \leftarrow X_{t, t+1}$. If the new value of $r_{1}$ is smaller than $x$, production is delayed, and so in the following period $t+1$, the value of $r_{1}$ will be given by $r_{1} \leftarrow r_{1}+$ $\left(X_{t, t+2}+X_{t+1, t+2}\right)$. If $r_{1}$ still remains smaller than $x$, we update its value in period $t+2$ to $r_{1} \leftarrow r_{1}+\left(X_{t-1, t+3}+X_{t, t+3}+X_{t+1, t+3}+X_{t+2, t+3}\right)$ (see figure 2 ).

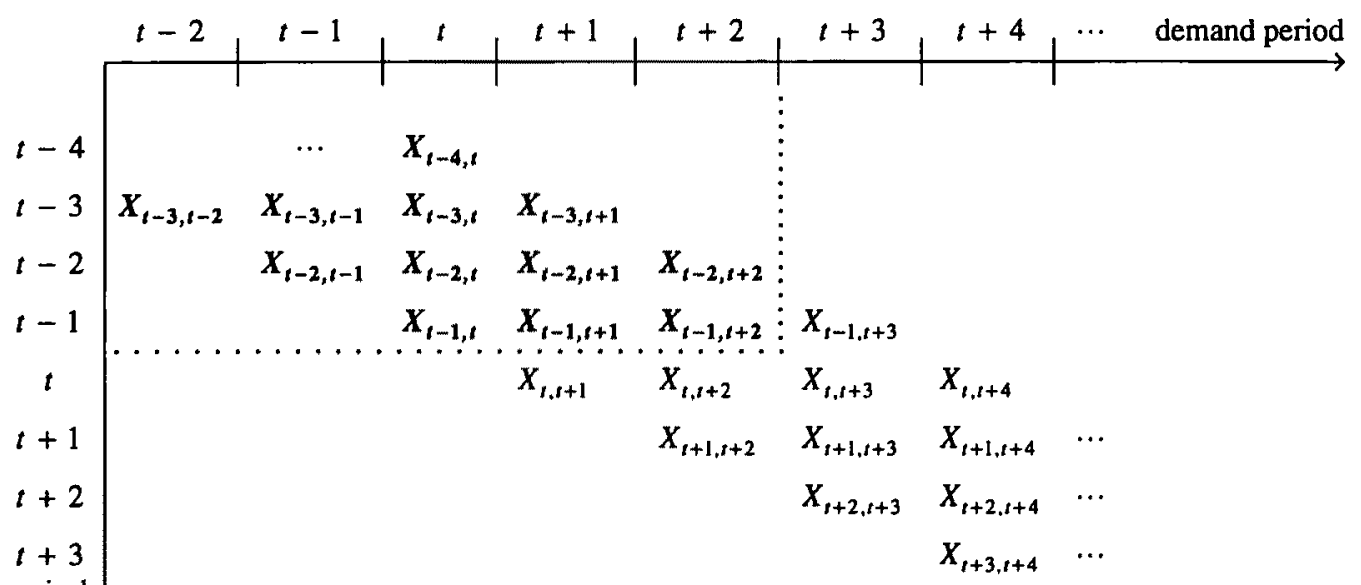

Figure 2. Example with $N=4$ and $T=3$. The known demand at the end of period $t-1$ to be produced during period $t$ is presented in bold. We assume that the last production took place $T$ periods ago.

From this analysis, it can easily be seen that if at the end of period $t+1$ or one of the later periods the value of $r_{1}$ is still smaller than $x$, then the value of $N$ stochastic variables has to be added to $r_{1}$ by the end of the next period. The probability that the sum of $i$ stochastic variables, whose value is added to $r_{1}$, is equal to $\ell$ is given by $b_{i \ell}$ as defined by (5). We will show that to determine the average costs per period for a given pair $(x, T)$ we only need to consider a finite recurrent Markov chain with states $(i, j) \in\{1,2, \ldots, T-1\} \times\{0,1, \ldots, x\}$ such that

$i \equiv$ number of periods since the last production period,

$j \equiv$ value of $r_{1}$.

The limitation on the number of possible states results from the fact that both the decision and the costs of that decision are the same for $r_{1}=x$ and $r_{1}>x$. Furthermore, 


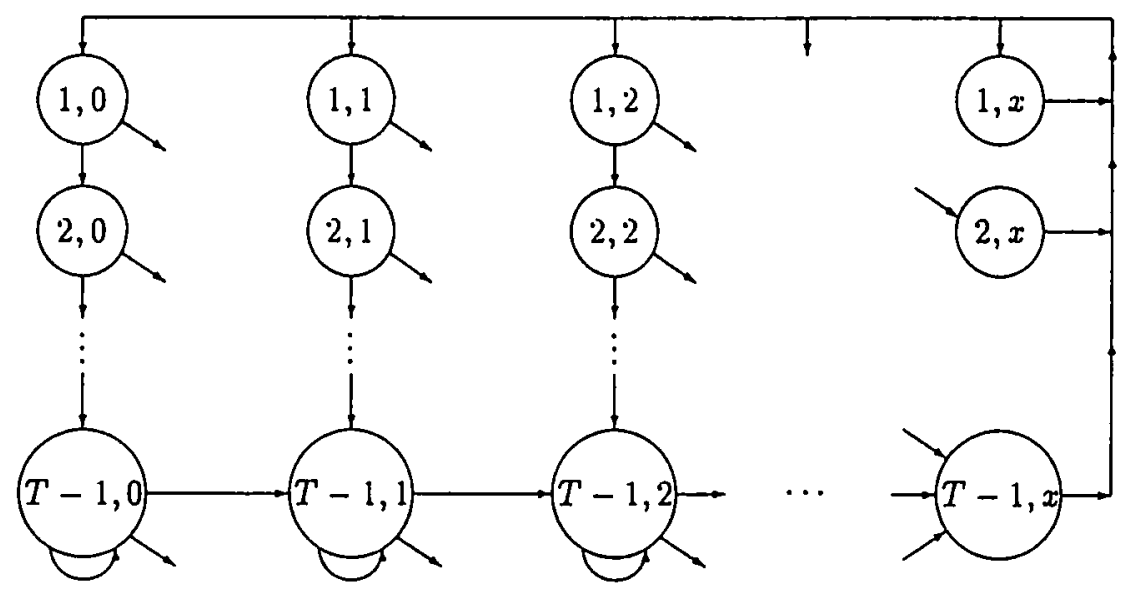

Figure 3. The states of the Markov chain for the $(x, T)$-rule with $T \geq 2$.

both the costs of an action and the transition probabilities for the component $r_{1}$ are the same for all periods except the $T-1$ first periods. In the case $T=1$, the time component is limited to $\{1\}$. Figure 3 gives as illustration of this Markov chain. In the above Markov chain, we have the following transition probabilities:

$$
\begin{array}{lll}
\mathcal{P}\{(i, x) \rightarrow(1, k)\}=b_{1, k} & 1 \leq i \leq T-1, & 0 \leq k \leq x-1, \\
\mathcal{P}\{(i, x) \rightarrow(1, x)\}=1-\sum_{k=0}^{x-1} b_{1, k} & & 1 \leq i \leq T-1, \\
\mathcal{P}\{(i-1, j) \rightarrow(i, k)\}=b_{i, k-j} & 2 \leq i \leq T-1, & j \leq k \leq x-1, \\
\mathcal{P}\{(i-1, j) \rightarrow(i, x)\}=1-\sum_{k=0}^{x-1-j} b_{i, k} & 2 \leq i \leq T-1, & 0 \leq j \leq x-1 .
\end{array}
$$

It remains to analyse the transition probabilities from a state $(T-1, j)$ to a state $(T-1, k)$, with $0 \leq j \leq x-1$ and $j \leq k \leq x$. Observe that a state $(T-1, j)$ can be visited not only during period $T-1$, but also in one of the later periods. In any case and following the discussion on the changes in the value of $r_{1}$ (see figure 2), from period $T-1$ onwards, the $r_{1}$ value is always updated with $N$ new stochastic variables. Hence, we can write

$$
\begin{array}{ll}
\mathcal{P}\{(T-1, j) \rightarrow(T-1, k)\}=b_{N, k-j} & j \leq k \leq x-1, \\
\mathcal{P}\{(T-1, j) \rightarrow(T-1, x)\}=1-\sum_{k=0}^{x-1-j} b_{N, k} & 0 \leq j \leq x-1 .
\end{array}
$$

In order to determine the average costs per period for a given pair $(x, T)$, the expected costs $c_{i j}$ in every possible state $(i, j)$ have to be calculated. Whenever $j<x$, there is no production and so only penalty costs $p j$ are incurred. In the remaining states $(i, x)$, set-up costs and usually also holding costs are charged. To avoid a complicated state space, the holding costs are paid immediately. Their expected value 
depends on the time passed since the last production period, that is, on the $i$-value of the state $(i, x)$. For $T>2$, it holds that if we have just produced in the previous period, the expected number of orders for future periods will not be as high as it would be after $T-1$ periods because some of the orders for these periods have already been produced (see figure 2). Thus, the expected costs in state $(i, j), 1 \leq i \leq T-1$ are given by

$$
c_{i j}= \begin{cases}p j & \text { if } 0 \leq j<x, \\ s+h \sum_{k=1}^{T-1} k e_{k+1}-h \sum_{k=1}^{T-i-1} k e_{k+i+1} & \text { if } j=x,\end{cases}
$$

with $e_{k}$ defined by (6).

Let $q_{i j}$ denote the expected amount of time spent in state $(i, j)$ between two arbitrary production periods. Note that only in the states $(T-1,0),(T-1,1), \ldots$, $(T-1, x-1)$ can we stay longer than one period. Clearly, the sum of the expected amount of time spent in all states gives the expected time between two production periods:

$$
T(x, T)=\sum_{i=1}^{T-1} \sum_{j=0}^{x} q_{i j} .
$$

On the other hand, the expected costs between two production periods are determined by

$$
C(x, T)=\sum_{i=1}^{T-1} \sum_{j=0}^{x} q_{i j} c_{i j}
$$

Hence, the average costs per period of the production rule associated with the pair $(x, T)$ are obtained by dividing (8) by (7):

$$
g(x, T)=\frac{C(x, T)}{T(x, T)} .
$$

Due to the special structure of the Markov chain, the values of $q_{i j}$ can be determined as follows. If $T \geq 3$, in the first period after a production period we enter state $(1, x)$ or one of the states $(1, j)$. Hence,

$$
q_{1 j}= \begin{cases}b_{1 j} & \text { if } 0 \leq j<x, \\ 1-\sum_{k=0}^{x-1} b_{1 k} & \text { if } j=x .\end{cases}
$$

For $T \geq 4$, we move from state $(i-1, k)$ to state $(i, j)$ with probability $b_{i, j-k}$ and enter state $(i, x)$ if the number of orders was less than $x$ in period $i-1$ and at least $x$ in period $i$. This yields for $2 \leq i \leq T-2$

$$
q_{i j}= \begin{cases}\sum_{k=0}^{j} q_{i-1, k} b_{i, j-k} & \text { if } 0 \leq j<x, \\ \sum_{k=0}^{x-1}\left[q_{i-1, k}-q_{i k}\right] & \text { if } j=x .\end{cases}
$$


Observe for $j=x$ that

$$
\begin{aligned}
q_{i x} & =\sum_{k=0}^{x-1} q_{i-1, k} \sum_{j=x-k}^{\infty} b_{i j} \\
& =\sum_{k=0}^{x-1} q_{i-1, k}\left(1-\sum_{j=0}^{x-k-1} b_{i j}\right) \\
& =\sum_{k=0}^{x-1} q_{i-1, k}-\sum_{k=0}^{x-1} \sum_{j=0}^{x-k-1} q_{i-1, k} b_{i j} .
\end{aligned}
$$

By rearranging the terms in the above expression, we obtain

$$
\begin{aligned}
q_{i x} & =\sum_{k=0}^{x-1} q_{i-1, k}-\sum_{k=0}^{x-1} \sum_{j=0}^{k} q_{i-1, j} b_{i, k-j} \\
& =\sum_{k=0}^{x-1} q_{i-1, k}-\sum_{k=0}^{x-1} q_{i, k},
\end{aligned}
$$

which is exactly the formula in (10) for $j=x$.

State $(T-1, j)$ can be visited during period $T-1$ but also in later periods We can move to this state from state $(T-2, k)$ as well as from a state $(T-1, k)$. If during the $N$ previous periods no deliveries have been ordered with this specific delivery date, then we stay in the same state with probability $b_{N 0}$. Furthermore, there is exactly one production period during one cycle. This means that if there is no production during the first $(T-2)$ periods, it will certainly take place in one of the later periods. Therefore, we have for $T \geq 3$

$$
q_{T-1, j}= \begin{cases}\sum_{k=0}^{j} q_{T-2, k} b_{T-1, j-k}+\sum_{k=0}^{j} q_{T-1, k} b_{N, j-k} & \text { if } 0 \leq j<x, \\ 1-\sum_{i=1}^{T-2} q_{i x} & \text { if } j=x .\end{cases}
$$

Observe that the first part of (11) is equivalent to

$$
q_{T-1, j}=\frac{\sum_{k=0}^{j} q_{T-2, k} b_{T-1, j-k}+\sum_{k=0}^{j-1} q_{T-1, k} b_{N, j-k}}{1-b_{N 0}}, 0 \leq j<x
$$

For the special case of $T=1$, the values of $q_{1 j}$ are given by

$$
q_{1 j}= \begin{cases}\frac{b_{N j}+\sum_{k=0}^{j-1} q_{1, k} b_{N, j-k}}{1-b_{N 0}} & \text { if } 0 \leq j<x \\ 1 & \text { if } j=x\end{cases}
$$


while for $T=2$, we have

$$
q_{1 j}= \begin{cases}\frac{b_{1 j}+\sum_{k=0}^{j-1} q_{1, k} b_{N, j-k}}{1-b_{N 0}} & \text { if } 0 \leq j<x, \\ 1 & \text { if } j=x .\end{cases}
$$

In order to find the pair $(x, T)$ that yields the lowest average costs per period, we can use two properties derived by Dellaert [5] which allow us to limit the number of pairs that have to be considered. In the next result, an upper bound on the optimal value of $x$ is established given a value of $T$. We refer to Dellaert [5] for a proof of this result.

PROPERTY 5.1

For a given value of $T$, the optimal value of $x$ satisfies

$$
x \leq\left\lfloor\frac{g(x, T)}{p}\right\rfloor+1,
$$

where $\lfloor z\rfloor$ denotes the largest integer not larger than $z \in \mathbb{R}$.

By combining this property with the fact that $x \geq 1$, we obtain an interval containing the optimal choice for $x$.

An immediate consequence of the above property is the following result.

\section{COROLLARY 5.2}

Let $g(y, T)$ be the average costs of the $(x, T)$-rule for an arbitrary pair $(y, T)$. Since $g(x, T) \leq g(y, T)$, the optimal value $x$ satisfies

$$
x \leq\left\lfloor\frac{g(x, T)}{p}\right\rfloor+1 \leq\left\lfloor\frac{g(y, T)}{p}\right\rfloor+1 .
$$

Moreover, for $T \leq 2$, the optimal value of $x$ satisfies relation (12) with equality.

Let us now introduce $q_{i j}(K)$ as the $q_{i j}$-value associated with the $(x, K)$-rule and let $p_{i j}(K)$ be the probability that between two production periods the state $(i, j)$ is visited, if there is no limitation on the state space in the time-direction. For these probabilities $p_{i j}(K)$, we thus allow $i \geq K$. It is not difficult to see that $p_{i j}(K)=q_{i j}(K)$ for $i \leq K-2$ and $0 \leq j \leq x$. For the remaining values of $i$, the probabilities $p_{i j}(K)$ are easily obtained by the corresponding transition probabilities. This implies for $K \geq 3$ and $0 \leq j \leq x-1$ that 


$$
p_{i j}(K)= \begin{cases}q_{i j}(K) & \text { if } 1 \leq i \leq K-2, \\ \sum_{\ell=0}^{j} p_{i-1, \ell}(K) b_{i, j-\ell} & \text { if } i=K-1, \\ \sum_{\ell=0}^{j} p_{i-1, \ell}(K) b_{N, j-\ell} & \text { if } i \geq K .\end{cases}
$$

For $K=2$, we define $p_{1 j}(K)=b_{1 j}$ and $p_{2 j}(K)=\sum_{\ell=0}^{j} p_{1 \ell}(K) b_{N, j-\ell}$ for every $0 \leq j$ $\leq x-1$.

Finally, let $\bar{c}_{K}$ denote the expected costs in the $K$ th period after the last production period, if the $(x, K+1)$-rule is used and there was no production during that period. Clearly,

$$
\bar{c}_{K}=p \frac{\sum_{i=0}^{x-1} i p_{K i}(K+1)}{\sum_{i=0}^{x-1} p_{K i}(K+1)}
$$

Since at the end of each review period our knowledge about the future required deliveries, although incomplete, only extends to the next $N$ periods, it is obvious that $T$ can not exceed $N$. However, in some cases it is possible to prove that the best choice for $T$ lies in the interval $[1, K]$, with $K \leq N$. The following result shows how such an improved upper bound $K$ on the optimal value of $T$ can be obtained.

\section{PROPERTY 5.3}

For a given value of $x$, the optimal value of $T$ is less than or equal to $K$ if for $K$ the following inequality holds:

$$
\left(\bar{g}-\bar{c}_{K}\right) \sum_{j=0}^{x-1} p_{K j}(K+1)<h K e_{K+1},
$$

where $\bar{g}$ is an arbitrary upper bound for the average costs $g(x, T)$ and $p_{K j}(K+1)$ and $\bar{c}_{K}$ are determined by (13) and (14), respectively.

\section{Proof}

We refer to Dellaert [5] for a proof of this result.

By combining properties 5.1 and 5.3, we can design an algorithm to find the optimal pair $(x, T)$. A description of such a procedure is given below.

\section{ALGORITHM 1}

Step 0. Set $g^{*}:=s$ and $x:=\lfloor s / p\rfloor+1$.

Step 1. Set $K:=1$ and determine the first $K$ value for which (15) holds.

Set $T:=K$, determine $g(x, T)$ by (9) and set $g^{*}:=g(x, T)$. 
Step 2. For the current value of $T$, determine the optimal $x$ value as follows. Repeat $x:=\left\lfloor g^{*} / p\right\rfloor+1$ and $g^{*}:=g(x, T)$ until the value of $x$ does not change. Then set $x:=x-1$ and compute $g(x, T)$.

Repeat this procedure until a further decrease of $x$ yields a higher $g(x, T)$ value.

Let $g^{*}$ be the minimum value of $g(x, T)$ obtained in this step.

If the corresponding $x$ value is different from the one at the beginning of this step, then go to step 3 with the new $x$ value. Otherwise, stop.

Step 3. For the current value of $x$, determine the optimal $T$ value as follows. Set $K:=T$ and decrease $K$ until inequality (15) no longer holds.

Set $T$ equal to the minimum value of $K$ which satisfies (15).

Set $T:=T-1$ and compute $g(x, T)$.

Repeat this procedure until a further decrease of $T$ yields a higher $g(x, T)$ value.

Let $g^{*}$ be the minimum value of $g(x, T)$ obtained in this step.

If the corresponding $T$ value is different from the one at the beginning of this step, return to step 2 with the new $T$ value. Otherwise, stop.

Observe that the above algorithm terminates when a further decrease of $x$ or $T$ yields higher average costs per period. Moreover, each time $g(x, T)$ has to be determined, it is not necessary to compute every $q_{i j}$ for $1 \leq i \leq T-1$ and $0 \leq j \leq x$ because most of these values are not affected by decreasing one of the two components, $x$ or $T$. The same occurs with respect to the probabilities $p_{i j}(K)$. Also, it is quite simple to obtain the $q_{i j}$-values from the $p_{i j}$-values and vice versa by relation (13).

\subsubsection{Refinement of the $(x, T)$-rule}

The relatively simple nature of the $(x, T)$-rule makes its understanding and application easy for practitioners. Clearly, since only the information about the demand for the first period is used, there is no guarantee that we will be following an optimal strategy in every period. In fact, if the orders for the next $T$ periods do not deviate from their expected value, the $(x, T)$-rule gives a nearly optimal action for each period of the planning horizon. However, in cases of unexpectedly high or low demand, the rule may select a non-optimal action. In such situations, a simple set of tests in which we estimate the future costs of various actions can be applied to give us an indication of when we have to deviate from the $(x, T)$-rule. In this subsection, we concentrate on refining the heuristic by means of four tests that can lead to cost improvements. These tests are meant to be applied at the end of each period on observing a certain sequence $\left(r_{1}, r_{2}, \ldots, r_{T}\right)$.

If the holding costs are relatively low, we may consider producing during a period for which $r_{1}<x$. In order to determine whether it is preferable to produce or not in such a situation, we need to compare the costs of producing during the current 
period with the costs of only producing in the next period. Observe that this comparison is only meaningful in those cases for which we know that production will certainly take place in the following period. This corresponds to having $r_{1}+r_{2} \geq x$ or $r_{1}+r_{2}<$ $x$ and at least new $\left(x-r_{1}-r_{2}\right)$ orders are placed during the current period to be delivered by the end of the next period.

Let $g^{*}$ denote the average costs $g(x, T)$ per period of the optimal pair $(x, T)$ and let $\bar{c}$ denote the expected costs of action $a=T$ assuming that no part of the demand for the next $T$ periods was already produced, i.e. $\bar{c}=s+h \sum_{i=1}^{T-1} i e_{i+1}$.

We can estimate the costs of the options described above by using more information than that contained in $r_{1}$, namely, the values of $r_{2}, \ldots, r_{T}$. It follows that

(i) Expected costs of taking action $a=T$ in the current period:

$$
\bar{c}+h \sum_{i=1}^{T-1} i\left(r_{i+1}-e_{i+1}\right)+h \max \left(0, x-r_{1}-r_{2}\right) .
$$

(ii) Expected costs of taking action $a=T$ in the next period:

$$
p r_{1}+\bar{c}+h \sum_{i=2}^{T-1}(i-1)\left(r_{i+1}-e_{i+1}\right)-g^{*} .
$$

Comparing (i) with (ii) yields the following test.

TEST 1. PROCEDURE SOONER

For a sequence $\left(r_{1}, r_{2}, \ldots, r_{T}\right)$ such that $r_{1}<x$ and $h \sum_{i=1}^{T-1}\left(r_{i+1}-e_{i+1}\right)$ $<p r_{1}-g^{*}-h \max \left(0, x-r_{1}-r_{2}\right)$, select action $a=T$.

The effect of having high holding costs can be dealt with in different ways. In some situations, it may be advantageous to postpone the production. If $r_{1} \geq x$ and we decide not to produce during this period, we will choose that action in the following period. As a result, we have the same options as in the previous test and the corresponding expected costs are identical except for the last term in $(i)$, which is excluded. Therefore, the second test is as follows.

\section{TEST 2. POSTPONE PRODUCTION}

For a sequence $\left(r_{1}, r_{2}, \ldots, r_{T}\right)$ such that $r_{1} \geq x$ and $h \sum_{i=1}^{T-1}\left(r_{i+1}-e_{i+1}\right)$ $>p r_{1}-g^{*}$, select action $a=0$.

A second way of dealing with high holding costs is to consider producing the known orders for less than $T$ periods. This action may be beneficial, for instance, when $r_{T}$ is large. Hence, we have to compare actions $a_{k}=T-k, k=1,2, \ldots, T-1$, with action $a=T$. Fixing $k$, the estimation of the costs involved in action $a_{k}$ is based 


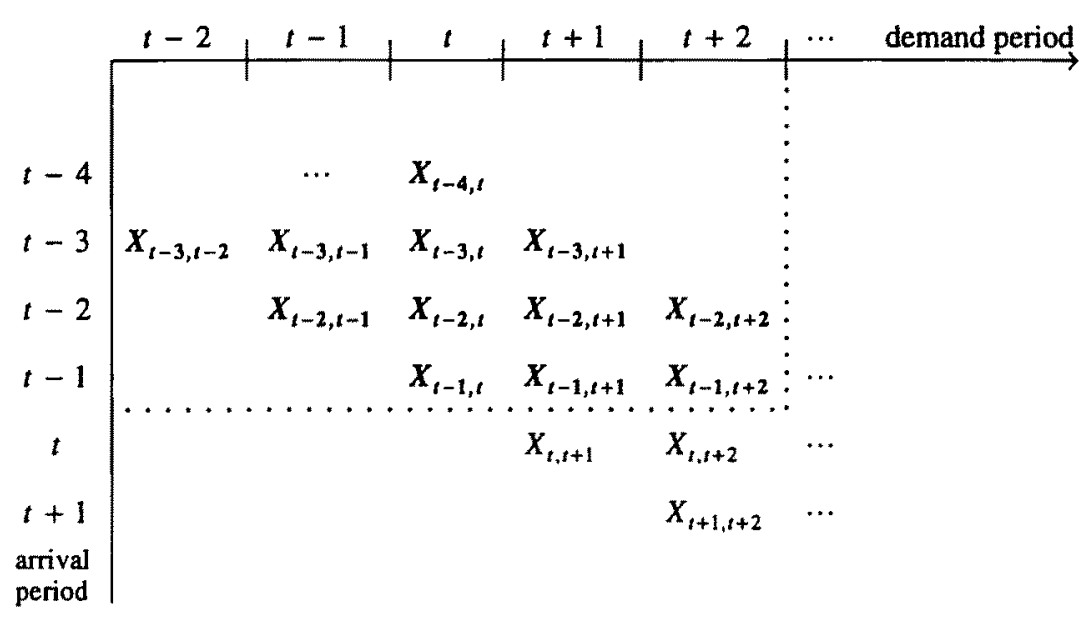

Figure 4. Example with $N=4$ and $a=3$. Orders produced during period $t$ are indicated in bold assuming that the last production took place $a$ periods ago. In periods $t+1$ and $t+2$, there is no production.

on the assumption that if we produce the required deliveries for $a_{k}$ periods, then during the next $a_{k}-1$ periods there will be no production.

Without loss of generality, we can drop the index $k$ and analyse the consequences of the above assumption. If $a \geq 2$ and production takes place during some period $t$, then the required deliveries for periods $t+1, \ldots, t+a-1$ that are ordered during periods $t, t+1, \ldots, t+a-2$ will be delivered too late. As a result, penalty costs have to be paid for these orders. Figure 4 illustrates an example of this situation.

The exact penalty costs are difficult to calculate since the future demands are unknown. However, we can replace them by their expected value during the first $a$ periods under the assumption that production only takes place during the first period and not in the next $a-1$ periods. This yields the following penalty function $p(a)$ :

$$
\begin{aligned}
p(a) & =p \mathcal{E}\left(\sum_{k=2}^{a} \sum_{i=2}^{k} \sum_{j=1}^{i-1} X_{j i}\right)=p \sum_{k=2}^{a} \sum_{i=2}^{k} \sum_{j=1}^{i-1} u_{i-j} \\
& =p \sum_{i=2}^{a}(a+1-i) \sum_{j=1}^{i-1} u_{j}, \quad a=2,3, \ldots, N .
\end{aligned}
$$

For $a=0$ and $a=1$, we have

$$
p(a)=0, a=0,1 .
$$

Proceeding with the derivation of a third test to improve the performance of the $(x, T)$-rule, we next give an overestimation of the costs of action $a_{k}=T-k$ with $k$ fixed $(1 \leq k \leq T-1)$. 
(iii) Expected costs of taking action $a_{k}=T-k$ in the current period and action $a=T, T-k$ periods later:

$$
\begin{gathered}
\left(\bar{c}-h \sum_{i=T-k}^{T-1} i e_{i+1}\right)+h \sum_{i=1}^{T-k-1} i\left(r_{i+1}-e_{i+1}\right)+p(T-k) \\
+\bar{c}+h \sum_{i=2}^{k}(i-1)\left(r_{T-k+i}-e_{T-k+i}\right)-(T-k) g^{*}
\end{gathered}
$$

Comparing (iii) with the expected costs of action $a=T$ leads to the following test.

TEST 3. PRODUCE THE DEMAND FOR LESS THAN $T$ PERIODS

Given $k(1 \leq k \leq T-1)$ and a sequence $\left(r_{1}, r_{2}, \ldots, r_{T}\right)$ such that $r_{1} \geq x$ and $\bar{c}+p(T-k)-h \sum_{i=T-k}^{T-1} i e_{i+1}<(T-k) g^{*}+h(T-k) \sum_{i=T-k}^{T-1}\left(r_{i+1}-e_{i+1}\right)$, select action $a_{k}=T-k$.

In case the above test holds for several values of $k$, we choose that $k$ for which the difference between the right-hand and left-hand side terms of the inequality is maximal.

If Test 3 indicates that we should produce the demand for less than $T$ periods, then it seems reasonable to compare that decision with the possibility of delaying the production. Although Test 2 can not be applied in this case, we can easily derive a fourth condition by comparing the costs in (iii) with the costs in (ii). This leads to the following test

TEST 4. POSTPONE PRODUCTION AS AN ALTERNATIVE TO PRODUCING FOR LESS THAN $T$ PERIODS

Given $k(1 \leq k \leq T-1)$ and a sequence $\left(r_{1}, r_{2}, \ldots, r_{T}\right)$ for which Test 3 holds, evaluate the following inequality:

$$
\begin{aligned}
\bar{c} & +p(T-k)+h \sum_{i=1}^{T-1}\left(r_{i+1}-e_{i+1}\right)-h \sum_{i=T-k}^{T-1} i e_{i+1}>p r_{1} \\
& +(T-k-1) g^{*}+h(T-k) \sum_{i=T-k}^{T-1}\left(r_{i+1}-e_{i+1}\right) .
\end{aligned}
$$

If the inequality holds, then select action $a=0$, otherwise select action $a_{k}=T-k$.

Having determined the optimal pair $(x, T)$ and the corresponding average costs $g^{*}=g(x, T)$, we can use the above four tests to check at the end of each period if we need to deviate from the $(x, T)$-rule on observing a certain sequence $\left(r_{1}, r_{2}, \ldots, r_{T}\right)$. The following algorithm indicates the order in which the tests should be applied. 


\section{ALGORITHM 2}

Step 0. If

$$
r_{1} \leq \frac{g^{*}+x-\sum_{i=1}^{T-1} e_{i+1}}{p+1}
$$

Then production does not take place;

$$
\text { GoTo step } 5
$$

Else GoTo step 1;

Step 1. If $r_{1} \geq x$ Then apply Test 3;

If the test holds Then GoTo step 4

Else GoTo step 2

Else GoTo step 3;

Step 2. Apply Test 2.

If the test holds Then delay the production

Else produce the demand for $T$ periods;

GoTo step 5;

Step 3. Apply Test 1.

If the test holds Then produce the demand for $T$ periods

Else delay the production;

GoTo step 5;

Step 4. Apply Test 4.

If the test holds Then delay the production

Else produce the demand for $T-k$ periods;

GoTo step 5;

Step 5. Stop.

For a given sequence $\left(r_{1}, r_{2}, \ldots, r_{T}\right)$ satisfying one of the tests described above, we can estimate the corresponding cost improvement by determining the probability of having the sequence $\left(r_{1}, r_{2}, \ldots, r_{T}\right)$ and then multiplying by the positive difference between the right-hand side term and the left-hand side term of the inequality in the test. Observe that the probability of having a certain sequence $\left(r_{1}, r_{2}, \ldots, r_{T}\right)$ is given by the product of the probabilities for each component $r_{i}$, with $i=1, \ldots, T$. In the case of $r_{1}$, we can easily use the values of $q_{i j}$ to obtain the corresponding probability. For the remaining components, it is not difficult to show that

$$
\mathcal{P}\left(r_{i}=\ell\right)=\sum_{J_{i, \ell_{i}}} \prod_{n=i}^{N} d_{n j_{n}}, \quad i=2, \ldots, T,
$$

with $J_{i, \ell_{i}}$ denoting the set of one-period demands $\left(j_{1}, j_{2}, \ldots, j_{n}\right)$ for which $\sum_{n=i}^{N} j_{n}=\ell_{i}$. Furthermore, an estimation of the total savings per period can be obtained by applying the above algorithm to each possible combination of values for $\left(r_{1}, r_{2}, \ldots, r_{T}\right)$ that has 
a probability greater than some small pre-specified constant $\varepsilon$. Finally, adding the gains produced by the tests and subtracting this sum from $g^{*}$ yields an estimation of the improved average costs per period.

\subsection{THE SILVER-MEAL APPROACH}

For a lot-sizing problem with a deterministic time-varying demand, Silver and Meal [23] proposed a simple sequential heuristic which consists of selecting the action that produces the (first local) minimum of the total relevant costs per unit of time. These costs are obtained by dividing the expected costs of an action by the number of periods involved in that action. In this section, a lot-sizing rule using the same criterion is derived for our problem.

Choosing action $a$ on observing a state $r \in R$ leads to the direct costs $q_{r}^{a}$ given by (2). However, also indirect costs will be involved during the first $a$ periods if we assume that during the next $a-1$ periods there will be no production. Clearly, this leads to penalty costs that are estimated by the function $p(a)$ defined in (16) and (17).

Hence, if state $r \in R$ is observed, the production rule consists in taking action $a^{\prime}$ given by

$$
a^{\prime}:=\arg \min \left\{\frac{q_{r}^{a}+p(a)}{\max \{1, a\}}: a \in A(r)\right\} .
$$

We can now use a similar dynamic programming algorithm to the one presented in section 4 to obtain the average costs per period. The iteration scheme in this case takes the form

$$
v_{n+1}(r)=q_{r}^{a^{\prime}}+\sum_{z \in R} P_{r z}^{a^{\prime}} v_{n}(z), \quad r \in R, n=0,1, \ldots
$$

\subsection{THE CYCLIC PRODUCTION RULE}

The cyclic production rule that is described next is the equivalent of the Period Order Quantity lot-sizing technique proposed by Gorham [8] in which the optimal production schedule in the case of constant demand requires a set-up every $T$ periods. We denote the production rule by $\pi$ and also assume that there is a set-up every $T$ periods, unless there is no demand. In addition, whenever production takes place, the required deliveries for the first $T$ periods are produced. In order to determine the average costs per period for this rule, we divide by $T$ the total expected costs during one cycle which consist of set-up costs, holding costs for the orders that are produced before their delivery date and penalty costs for the orders that will be finished after their delivery date. Therefore, we choose the value of $T \in\{1,2, \ldots, N\}$ for which $g_{\pi}$ is minimal, with $g_{\pi}$ given by

$$
g_{\pi}=\frac{s\left(1-\left(b_{N 0}\right)^{T}\right)+h \sum_{i=1}^{T-1} i e_{i+1}+p(T)}{T}
$$


and $p(T)$, the function defined by (16) and (17). Observe that the term $\left(1-\left(b_{N 0}\right)^{T}\right)$ gives the probability that there is demand for at least one of the next $T$ periods.

In cases with relatively stable demand patterns, it is expected that a cyclic production rule like the one described above performs suitably.

\section{Numerical results}

In order to study the performance of the competing lot-sizing rules presented in the previous section, two types of experiments were conducted over a range of test problems. In the first group of tests, the demand pattern was fixed while the cost parameters changed. In the second group of tests, the demand parameters varied and the cost structure was held fixed.

We will start with a very simple set of examples for which demand follows a binary distribution. Although such a demand pattern is not likely to be observed in practice, it has the advantage that for relatively small values of $N$, the dimension of the state space is not too large and so we can apply the dynamic programming algorithm of section 4 to obtain the optimal average costs. Consequently, each heuristic can be compared with the optimal production policy. Moreover, the average costs associated with the Silver-Meal-like strategy can also be computed directly.

Table 1 summarizes the results obtained by assuming that the demand of each group $i$ of customers follows the same binary distribution with parameter $d$, that is, $d_{i 0}=1-d$ and $d_{i 1}=d$ for $i=1, \ldots, N$. As a result, the probabilities $b_{i \ell}$ in (5) are easily determined by $b_{i \ell}=\left(\begin{array}{c}i \\ \ell\end{array}\right)(1-d)^{i-\ell} d^{\ell}$ if $1 \leq \ell \leq i \leq N$ and $b_{i \ell}=0$ otherwise. For each choice of the demand parameter $d$, two tests were performed where two of the cost parameters were held fixed while the third one changed. By proceeding in this way, we could examine the effect of the cost structure on the behaviour of the lot-sizing rules. The choice of the cost parameters was taken as follows: the penalty costs were always larger than the holding costs but smaller than the set-up costs. These latter costs were at least twice as big as the penalty costs and in some cases, the proportion increased to $16: 1$. Finally, the penalty costs were at most three times larger than the holding costs. The results reported in table 1 correspond to tests where the values of $s, h$ and $p$, although set arbitrarily, followed the above criteria. They represent the differences in the three types of costs that are commonly observed in many practical situations.

The first column in table 1 refers to the number of each test problem. The second column indicates the maximum lead time $(N)$, while the third column gives the value of the demand parameter $(d)$. The following three columns present the cost structure selected in each test, that is, the set-up costs $(s)$, the holding costs $(h)$ and the penalty costs $(p)$. Column 7 contains the optimal average costs per period (OPT). In columns 8 and 9 , both the optimal pair $(x, T)$ and the corresponding average costs per period $g(x, T)$ are indicated. The results of the application of the four tests described in subsection 5.1.1 are presented in columns 10 and 11. The cost improvement 
Table 1

Results with binary demand.

\begin{tabular}{rllrrrrrrrrrrr}
\hline 1 & 2 & 3 & 4 & 5 & 6 & 7 & 8 & 9 & 10 & 11 & 12 & 13 & 14 \\
Test & $N$ & $d$ & $s$ & $h$ & $p$ & \multicolumn{1}{c}{ OPT } & $(x, T)$ & $g(x, T)$ & $\Delta$ & $\bar{g}(x, T)$ & $S M$ & $T$ & CYC \\
\hline 1 & 4 & 0.25 & 8 & 1 & 3 & 3.7147 & $(2,3)$ & 3.7326 & 0.0173 & 3.7153 & 3.7173 & 3 & 4.1655 \\
2 & 4 & 0.25 & 8 & 2 & 3 & 3.9871 & $(2,2)$ & 4.0219 & 0.0250 & 3.9969 & 4.0723 & 3 & 4.7245 \\
3 & 4 & 0.50 & 6.5 & 1 & 3 & 4.5357 & $(2,2)$ & 4.5392 & 0.0008 & 4.5384 & 4.5392 & 2 & 4.7373 \\
4 & 4 & 0.50 & 16 & 1 & 3 & 8.1705 & $(3,3)$ & 8.1965 & 0.0239 & 8.1725 & 8.1793 & 3 & 8.4987 \\
5 & 4 & 0.75 & 9.75 & 1 & 3 & 7.0425 & $(3,2)$ & 7.0451 & 0.0014 & 7.0438 & 7.0445 & 2 & 7.1249 \\
6 & 4 & 0.75 & 24 & 1 & 3 & 12.6002 & $(5,3)$ & 12.6125 & 0.0086 & 12.6040 & 12.6054 & 3 & 12.7500 \\
7 & 5 & 0.50 & 90 & 5 & 10 & 42.0968 & $(5,3)$ & 42.3478 & 0.1630 & 42.1849 & 42.7197 & 3 & 44.9991 \\
8 & 5 & 0.50 & 90 & 5 & 15 & 46.7550 & $(4,3)$ & 47.0620 & 0.2215 & 46.8405 & 46.8801 & 3 & 48.3324 \\
9 & 5 & 0.30 & 140 & 8 & 15 & 50.9724 & $(4,3)$ & 51.3558 & 0.1373 & 51.2184 & 51.5278 & 5 & 55.5962 \\
10 & 5 & 0.30 & 140 & 8 & 24 & 57.9336 & $(3,3)$ & 58.0856 & 0.0611 & 58.0246 & 58.1479 & 4 & 62.5721 \\
11 & 6 & 0.40 & 50 & 1 & 3 & 16.5934 & $(6,4)$ & 16.6298 & 0.0258 & 16.6040 & 16.7010 & 5 & 17.2000 \\
12 & 6 & 0.40 & 50 & 2 & 3 & 18.0522 & $(7,3)$ & 18.2419 & 0.1061 & 18.1358 & 18.5207 & 5 & 19.6000 \\
\hline
\end{tabular}

obtained is denoted by $\Delta$ and the new estimation of the average costs is given by $\bar{g}(x, T)=g(x, T)-\Delta$. Column 12 contains the average costs produced by the SilverMeal-like strategy. Finally, in columns 13 and 14, the results of the cyclic rule can be found, namely, the length of each cycle $(T)$ and the corresponding average costs per period (CYC).

The choice of values for $N$ between 4 and 6 results in state spaces whose dimension is still within reasonable limits. However, both the computation times and the storage requirements increase considerably with $N$ in the calculation of the average costs of the optimal policy and the Silver-Meal-like rule. On the contrary, both the $(x, T)$-rule and the cyclic production strategy require low computational effort.

From the above table, we can observe that although in half of the examples the Silver-Meal-like strategy performs better than the $(x, T)$-rule, the refinement of the latter results in costs that are below those of the Silver-Meal-like strategy. In fact, the lowest average costs are obtained in every case by applying the four tests included in algorithm 2 to the best pair $(x, T)$. This produces average costs very close to the optimal average costs, with a variation between $0.02 \%$ and $0.48 \%$. The cyclic production rule is clearly the worst strategy. This is caused by the fact that in this rule, production takes place too often even when $r_{1}=0$. A closer analysis of the Silver-Meal strategy showed that production occurs less frequently than in the optimal policy: production is delayed too long and when a set-up is paid, the amount produced is too large.

The influence of the set-up costs can be observed in tests $3,4,5$ and 6 . An increase in $s$ also results in an increase in the values of $x$ and $T$. This is natural since it becomes more expensive to start the production. Therefore, we wait until a larger 
number of orders for the next period is gathered and we manufacture the known demand also for more periods. In the cyclic rule, the same occurs with respect to the production cycle which increases from 2 to 3 periods. The effect caused by changes in the penalty costs is shown in tests $7,8,9$ and 10 . As one would expect, when late orders become more expensive, production starts earlier, but the number of periods covered by each setup remains the same since penalty costs are not incurred when we manufacture. However, in the cyclic rule the value of $T$ may decrease as indicated by example 10. Finally, in tests $1,2,11$ and 12 , the results of variations in the holding costs can be examined. An increase in $h$ affects the value of $T$ in the $(x, T)$-rule but does not produce any change in the $T$-value of the cyclic strategy. It seems natural that higher holding costs cause a decrease in the number of periods for which demand is produced since untimely deliveries for periods that are further away lead to larger costs. Regarding the value of $x$, either it remains unchanged like in test 2 or increases as in test 12. The amplitude of the setup costs also influences the $x$-value in this case. Observe that in test 2, the set-up costs are four times larger than the holding costs, while in test 12 they are 25 times higher. Hence, in this last case, an increase in $h$ also delays the next set-up.

The following examples are constructed by assuming demand patterns that are closer to real situations than the cases discussed above. We have chosen the binomial and the geometric distributions to illustrate the behaviour of the competing production rules. Furthermore, the next problems belong to the second group of tests for which the cost structure was held fixed and the demand parameters changed. The choice of the costs $s, h$ and $p$ was arbitrary, but followed the same criteria as in the binary demand examples, i.e. $s>p>h$. The main difference occurs in the $s$-value, which is considerably larger compared to $h$ and $p$. This choice is due to the demand patterns selected in which a higher number of orders is present. Due to the large dimension of the state spaces involved, the average costs of the Silver-Meal-like rule are obtained by means of simulation. For a fixed set of values for the parameters $N, s$, $h$ and $p$, and a given demand pattern, the average costs per period for each lot-sizing rule are determined by considering different coefficients of variation for the demand. If $X$ is a random variable denoting the amount of product ordered by the customers of a group during a certain period, then the quantity $c v=\sqrt{\operatorname{var}(X)} / \mathcal{E}(X)$ can be used to measure the variability of the demand. By assuming the same demand distribution for every group of customers and trying different parameter combinations in such a way that the expected amount ordered per period is always the same, we can analyse the behaviour of the heuristics for different coefficients of variation $(c v)$.

Figure 5 depicts the results obtained for a set of test problems with a maximum lead time of $N=4$ periods, set-up costs $s=75$, holding costs $h=1$ and penalty costs $p=2$. The demand in every group of customers follows a binomial distribution with mean $n \rho$ such that

$$
d_{i j}=\left(\begin{array}{c}
n \\
j
\end{array}\right) \rho^{j}(1-\rho)^{n-j}, \quad i=1, \ldots, N,
$$

with $j=0, \ldots, n, n>0$ and $0<\rho<1$. 


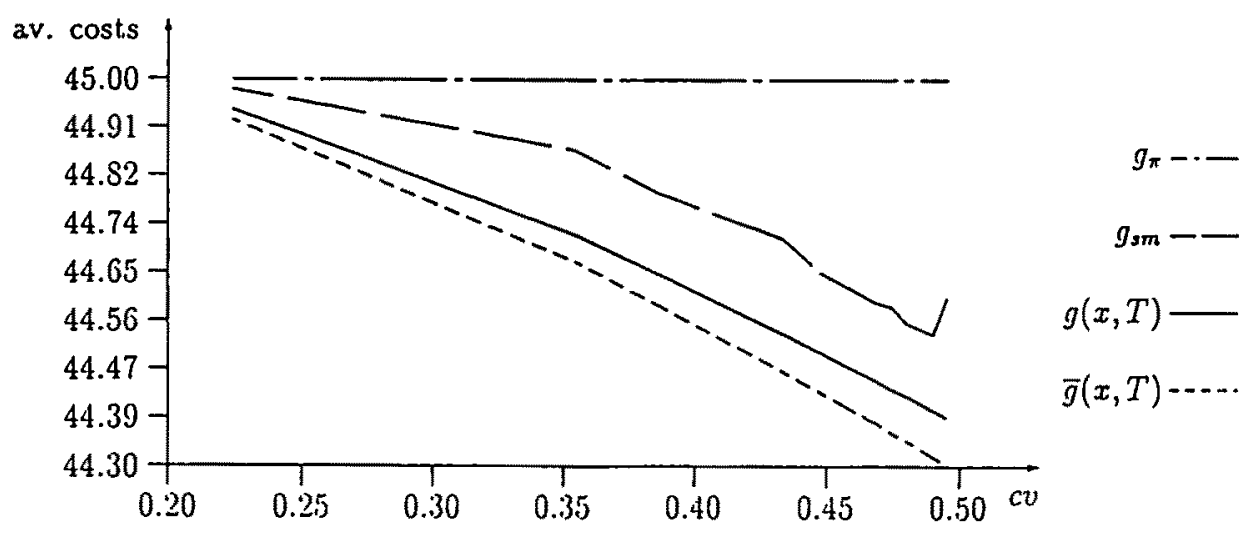

Figure 5. Binomial demand with mean 4.

We first fixed the mean number of ordered units of product at $n \rho=4$ and combined $n \in[5,200]$ with $\rho \in[0.02,0.80]$ in such a way that for each test problem and each group of customers $n \rho=4$. As a result, $c v$ lies in the interval [0.224, 0.495].

In figure 5 , we can observe the progress of the average costs per period in the cyclic production rule $\left(g_{\pi}\right)$, in the Silver-Meal-like strategy $\left(g_{s m}\right)$ and in the $(x, T)$ rule $(g(x, T))$. In each test problem, the latter rule gives the optimal pair $(23,2)$ to which the refinement discussed in subsection 5.1.1 is applied in order to obtain improved average costs denoted by $\bar{g}(x, T)$. As is expected, the cyclic rule is not affected by the coefficient of variation. As the relative variability of the demand increases, the average costs in the remainder strategies decrease gradually except for the Silver-Meal-like rule in the neighbourhood of $c v=0.5$. Since demand is binomial, by (18) it can be shown that each $r_{i}$ with $2 \leq i \leq N$ also follows a binomial distribution. An increasing coefficient of variation corresponds to an increase in the maximum number of orders that can be placed by every group of customers (i.e. the parameter $n$ of the distribution) and to a decrease in the parameter $\rho$. Consequently, the distribution of the components of the order state vector become skewed to the left and the larger $c v$ is, the more pronounced is the skewness. Furthermore, the probabilities $\mathcal{P}\left(r_{i}=\ell_{i}\right)$ also decrease with the coefficient of variation. The combination of these elements - variability, skewness and smaller probabilities for the orders in $r_{i}$-yields lower average costs per period since savings in holding costs are obtained as a result of fewer orders. The Silver-Meal-like strategy slightly deviates from this pattern, but its more unstable behaviour is probably due to the fact that simulation is used to determine the average costs. The refinement of the $(x, T)$-rule gives in all cases the best results and this becomes more evident as the relative variability of the demand increases. In many situations, cost savings are achieved by deviating from the pair $(x, T)=(23,2)$. This means that for some sequences $\left(r_{1}, r_{2}\right)$ such that $r_{1} \geq 23$, production is postponed. For other sequences with $r_{1}<23$, it proves to be cheaper to manufacture the orders in $r_{1}$ and $r_{2}$ instead of delaying the production. An increase in the demand 


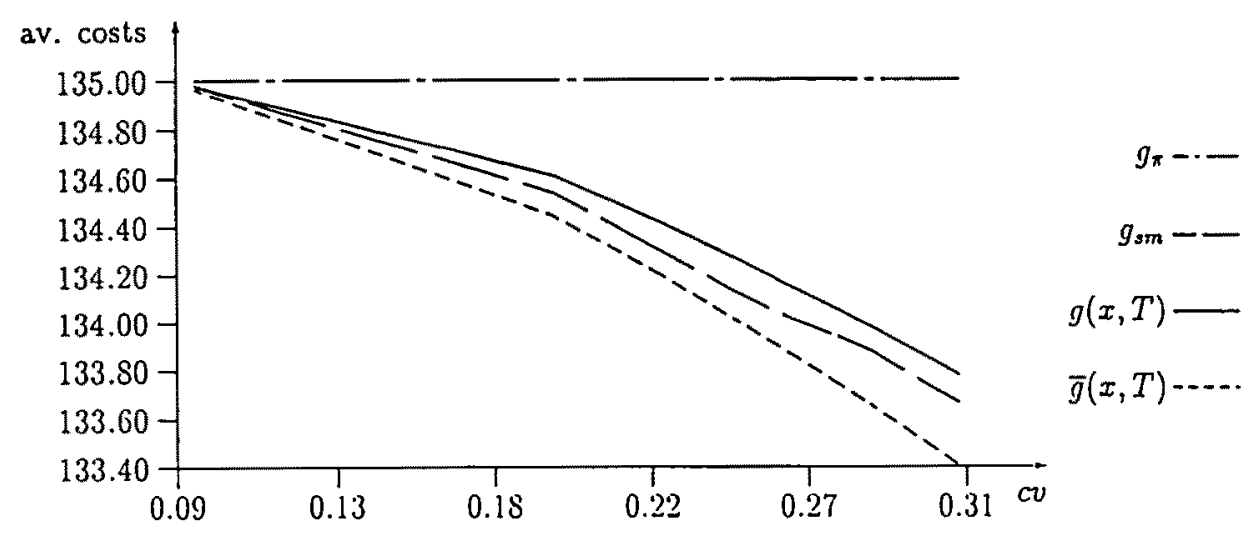

Figure 6. Binomial demand with mean 10.

variability raises the number of deviations from the pair $(x, T)$, namely, more sequences satisfy the inequality in test 2 . For this reason, the average costs tend to diminish.

Enlarging the expected number of orders from 4 to 10 leads to parameter variations in the intervals $[11,200]$ and $[0.05,0.91]$ for $n$ and $\rho$, respectively. Consequently, $c v \in[0.095,0.308]$. The results obtained with $N=4, s=180, h=2$ and $p=3$ are presented in figure 6 . Here, the Silver-Meal-like strategy is more stable and performs better than the $(x, T)$-rule which gives the optimal pair $(45,2)$. However, the refinement of this rule yields lower costs and the cost improvement obtained is higher for larger values of $c v$. As can also be observed in figure 6 , the variation in the costs $g(x, T), \bar{g}(x, T)$ and $g_{s m}$ are very small, never exceeding $1.2 \%$ of the costs of the cyclic rule. These variations increase with $c v$ due to the behaviour of the distributions for the number of orders which have a similar form to those of the first example.

Figure 7 reports on the results of several experiments conducted by assuming a geometric demand distribution. Like in the binomial case, we also fix the mean number of orders per period in, say, $c$ units. Then we consider the demand of each group of customers to follow a geometric distribution whose domain is shifted to the right so that different parameter variations leading to the same expectation are possible. This means that for each $i=1, \ldots, N$, we have

$$
d_{i j}=(1-\alpha) \alpha^{j-k}, \quad j=k, k+1, \ldots,
$$

with $0 \leq k<c, \alpha(1-\alpha)^{-1}+k=c$ and $0<\alpha<1$.

Figure 7 depicts the results obtained by taking $c=8$, a maximum lead time of $N=4$ periods and the following cost structure: $s=200, h=2$ and $p=3.5$. The values of $k$ and $\alpha$ lie in the intervals $[0,7]$ and $[1 / 2,8 / 9]$, respectively. The geometric distribution is characterized by a wider range of variability as compared to the previous examples. This is confirmed by a larger distance between the average costs 


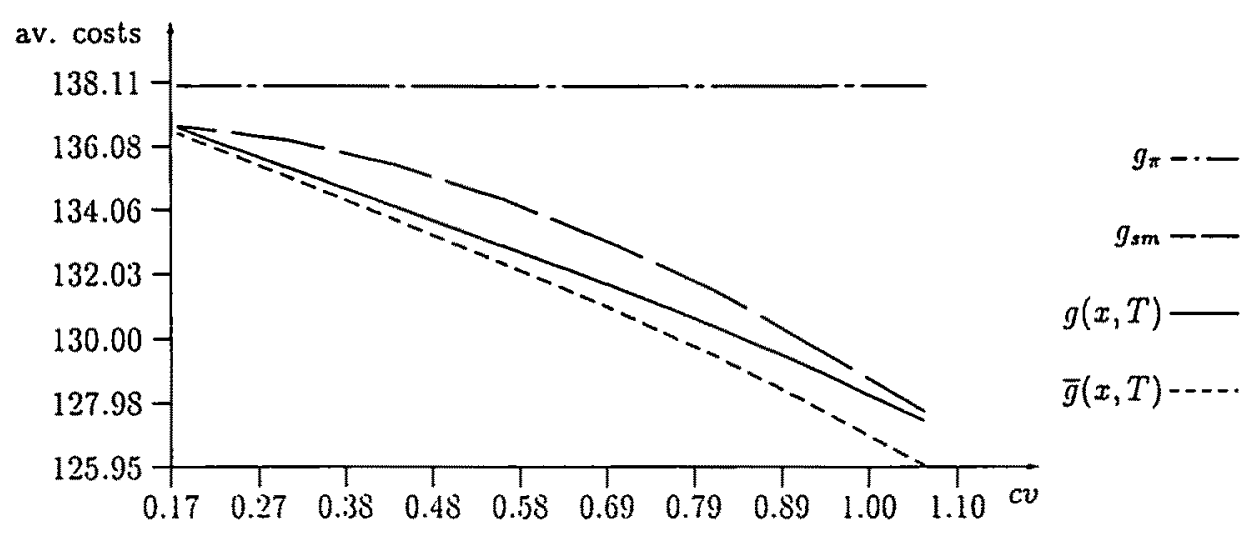

Figure 7. Geometric demand with mean 8.

of the cyclic rule and the costs of the other rules. Even when demand is almost deterministic, this difference is already significant and with the increase of $c v$, it almost reaches $9 \%$. The $(x, T)$-rule shows a better performance than the Silver-Meallike strategy in all test problems. The best choice for the value of $x$ varies between 40 and 37 , while $T$ always stays at 2 . This means that not only savings in holding costs are obtained as a result of the increasing skewness to the left just like in the binomial examples, but also lower penalty costs are incurred. The savings in the penalty costs are reflected in the optimal $x$-value and are an outcome of the shifts caused by the parameter $k$. Fixing the mean demand at 8 implies that the minimum number of orders that can be placed by each group of customers (i.e. $k$ ) gradually decreases from 7 to zero. Hence, it becomes more probable to have fewer orders for the first period and so it will be cheaper to take a set-up when $r_{1}$ is small. Regarding the impact of the refinement applied to each best $(x, T)$ pair, it can be seen that it is also stronger compared to the binomial case. The higher relative variability of the demand leads to more deviations from the pair $(x, T)$ and thus to larger savings. The deviations occur mainly in those sequences $\left(r_{1}, r_{2}\right)$ such that $r_{1} \geq x$. Production is then delayed or, instead of manufacturing the demand for $T=2$ periods, only the orders in $r_{1}$ are produced.

From the numerical experiments conducted, it appears that the $(x, T)$-rule combined with the improvements provided by the tests of subsection 5.1.1 gives the lowest average costs per period among all the heuristics. The cyclic production rule is clearly not suitable for situations of demand variability, as demonstrated by the tests. This is not surprising due to the nature of the rule. The Silver-Meal-like strategy has in some cases lower average costs compared to the $(x, T)$-rule even if the differences are not very high. Although the refinement of the $(x, T)$-rule seems to give the best results, the computation times depend on the number of different values for $\left(r_{1}, \ldots, r_{T}\right)$ that are considered. A way of avoiding too large computation times is to pre-specify a smaller precision $\varepsilon$ while selecting these values. Of course, a decrease in the time will be obtained at the expense of a smaller cost improvement. 
Concerning the practical problem observed in the company which motivated our research, it was observed for most of the product types that by choosing a proper unit size for the steel pipes, the demand could be modelled by a geometric distribution. The choice of the unit size determined the number of categories into which orders could fall. Clearly, if a very large scale was used, all orders would belong to the same category and a binary demand pattern could be applied. Although this aggregation would decrease the quality of the solutions, the computation time, on the other hand, would be reduced.

Our model was integrated in the operations control level of the hierarchical production planning system used by the company. The detailed decisions produced by the lot-sizing rules for individual items proved to be of great assistance to the management, since by capturing the uncertainties in the demand they provided the necessary feedback to evaluate the quality of the aggregate planning decisions.

\section{Conclusions}

In this paper, we addressed a stochastic lot-sizing problem inspired by a Dutch manufacturer working in the make-to-order sector. The main elements of our problem combine highly uncertain demand with fixed delivery dates for the customer orders and no possibility for holding safety stocks. The modelling approach consisted of a Markov Decision Process, for which a dynamic programming algorithm was presented in order to determine the optimal policy and the corresponding long-run average costs. In general, this procedure requires a substantial amount of computation and data storage due to the large dimension of the state space. In order to obtain approximations of the optimal average costs, three lot-sizing rules were presented. The first is a simple strategy called the $(x, T)$-rule where the known orders for the next $T$ periods are manufactured if the demand for the current period is at least $x$ units. The performance of this rule can be improved by applying a simple set of tests. The second lot-sizing rule is a Silver-Meal-like strategy where an estimation of the average costs per period is obtained for the best action associated with each order state vector. The drawback of having to use dynamic programming to determine the average costs can easily be overcome in this case by means of simulation. Finally, the third lot-sizing strategy is a fixed cycle production rule which is more appropriate for situations with relatively stable demand patterns. From the numerical experiments, we could learn that the performance of both the $(x, T)$-rule and the Silver-Meal rule is affected by the level of variability of the demand. The improvement of the $(x, T)$ rule appears to provide the best average costs. This strategy should be particularly attractive to practitioners due to its simple nature and also due to the possibility of determining the average costs analytically. Comparisons with the optimal average costs for binary demand distributions suggest that approximate solutions with the $(x, T)$-rule and the Silver-Meal-like strategy are very good. However, for more realistic demand patterns, such comparisons are no longer possible since the optimal 
policy is not available. In those cases, the evaluation of the quality of the lot-sizing rules should be made by comparison with a lower bound, preferably a good lower bound, on the optimal average costs. This seems to be an interesting topic for future research. At the present stage, capacity constraints are disregarded by our model. However, due to the good results obtained with the $(x, T)$-rule, we could try to follow a similar approach to the one used by Van Nunen and Wessels [27]. This would consist of first applying the lot-sizing rule and then incorporating the capacity requirements by means of a capacity adapting procedure. Another possibility would be to adapt directly the lot-sizing rules derived in this paper. In fact, we are presently investigating extensions of the three heuristics (Dellaert and Melo [6]) to situations with constant available capacity over the planning horizon. From the preliminary results obtained so far, the extension of the $(x, T)$-rule appears to perform rather well, especially in situations with tight capacity limits. However, the calculation of the average costs becomes more complex due to the fact that it may not always be possible to produce the demand for $T$ periods completely. Finally, the combination of make-to-order with make-to-stock in one single model also seems worth investigating.

\section{Acknowledgements}

The authors would like to thank the editor and two anonymous referees for their helpful comments on an earlier version of this paper.

\section{References}

[1] S. Axsäter, Evaluation of lot-sizing techniques, Int. J. Prod. Res. 24(1986)51-57.

[2] R. Bellman, Dynamic Programming (Princeton University Press, Princeton, 1957).

[3] A.B. Cruickshanks, R.D. Drescher and S.C. Graves, A study of production planning in a job shop environment, Manag. Sci. 30(1984)368-380.

[4] N.P. Dellaert, Multi-item production control for production to order, Eng. Costs Prod. Econ. 17(1989)167-173.

[5] N.P. Dellaert, Production to order: Models and rules for production planning, in: Lecture Notes in Economics and Mathematical Systems 333 (Springer, Berlin, 1989).

[6] N.P. Dellaert and M.T. Melo, Heuristic procedures for stochastic lot-sizing with capacity constraints, Technical Report TI-93-222, Tinbergen Institute, Rotterdam, The Netherlands (1993).

[7] E.V. Denardo and C.-Y. Lee, Error bound for the dynamic lot size model with backlogging, Ann. Oper. Res. 28(1991)213-228.

[8] T. Gorham, Dynamic order quantities, Prod. Inventory Manag. 9(1963)75-79.

[9] S.C. Graves, A.H.G. Rinnooy Kan and P.H. Zipkin (eds.), Logistsics of Production and Inventory, Handbooks in Operations Research and Management Science (Elsevier Science, 1993).

[10] L.C. Hendry, A decision support system to manage delivery and manufacturing lead times in make-to-order companies, Ph.D. Thesis, University of Lancaster (1989).

[11] L.C. Hendry and B.G. Kingsman, Production planning systems and their applicability to maketo-order companies, Euro. J. Oper. Res. 40(1989)1-15.

[12] L.C. Hendry and B.G. Kingsman, Job release: Part of a hierarchical system to manage lead times in make-to-order companies, J. Oper. Res. Soc. 42(1991)871-883. 
[13] J. Hoey, B.R. Kilmartin and R. Leonard, Designing a material-requirements-planning system to meet the needs of low-volume, make-to-order companies (with case study), Int. J. Prod. Res. 24(1986)375-386.

[14] H.A. Ten Kate, Order acceptance strategies and lead times, Paper presented at the 8th Int. Working Seminar on Production Economics, Igls, Austria (1994).

[15] B.G. Kingsman, L. Worden, L.C. Hendry, A. Mercer and E. Wilson, Integrating marketing and production planning in make-to-order companies, Int. J. Prod. Econ. 30-31(1993)53-66.

[16] C. Lecomte and P. Dejax, A decision support system for the production control of a make-to-order workshop, Paper presented at the 1st IFORS Specialized Conf. on Decision Support Systems, Bruges, Belgium (1991).

[17] R.E. Markland, K.H. Darby-Dowman and E.D. Minor, Coordinated production scheduling of make-to-order manufacturing, Euro. J. Oper. Res. 45(1990)155-176.

[18] L. Mattsson, J. Olhager, P. Ovrin and B. Rapp, Computerization of manufacturing planning and control systems: Minicomputer-based systems 1980-1987, Eng. Costs Prod. Econ. 17(1989) 71-78.

[19] D. McAreavey, J. Hoey and R. Leonard, Designing the closed loop elements of a material requirements planning system in a low-volume, make-to-order company (with case study), Int. J. Prod. Res. 26(1988)1141-1159.

[20] A. Odoni, On finding the maximal gain for Markov decision processes, Oper. Res. 17(1969) 857-860.

[21] M.A. Quaddus, A generalised method of optimal due date assignment by linear programming, J. Oper. Res. Soc. 38(1987)353-359.

[22] A. Seidmann and M.L. Smith, Due date assignment for production systems, Manag. Sci. 27(1981) $571-581$.

[23] E.A. Silver and H.C. Meal, A heurtsitic for selecting lot size requirements for the case of a deterministic time-varying demand rate and discrete opportunities for replenishment, Prod. Inventory Manag. 14(1973)64-74.

[24] E.A. Silver and R. Peterson, Decision Systems for Inventory Management and Production Planning, 2nd Ed. (Wiley, New York, 1985).

[25] H.C. Tijms, Stochastic Modelling and Analysis - A Computational Approach (Wiley, New York, 1986).

[26] N. Tobin, A. Mercer and B.G. Kingsman, A study of small subcontracting and make-to-order firms in relation to quotation for orders, Int. J. Oper. Prod. Manag. 6(1988)46-59.

[27] J.A.E.E. van Nunen and J. Wessels, Multi-item lot size determination and scheduling under capacity constraints, Euro. J. Oper. Res. 2(1978)36-41.

[28] H.M. Wagner and T.M. Whitin, Dynamic version of the economic lot size model, Manag. Sci. 5(1958)89-96.

[29] W.I. Zangwill, A deterministic multi-period production scheduling model with backlogging, Manag. Sci. 13(1966)105-119. 